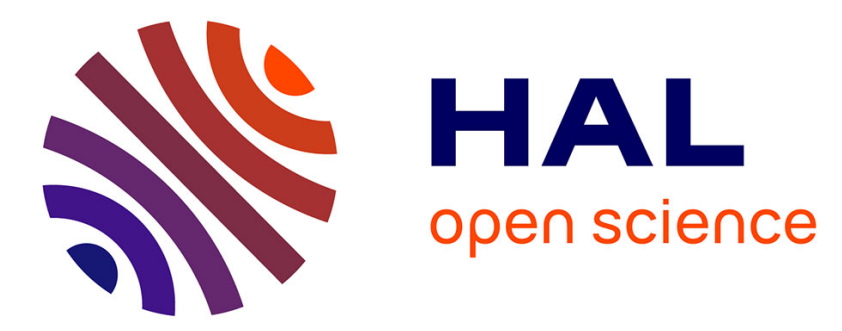

\title{
Review on single track vehicle and motorcycle simulators
}

Lamri Nehaoua, Hichem Arioui, Said Mammar

\section{To cite this version:}

Lamri Nehaoua, Hichem Arioui, Said Mammar. Review on single track vehicle and motorcycle simulators. 19th Mediterranean Conference on Control \& Automation (MED 2011), Jun 2011, Corfu, Greece. pp.940-945, 10.1109/MED.2011.5983083 . hal-00593407

\section{HAL Id: hal-00593407 \\ https://hal.science/hal-00593407}

Submitted on 15 May 2011

HAL is a multi-disciplinary open access archive for the deposit and dissemination of scientific research documents, whether they are published or not. The documents may come from teaching and research institutions in France or abroad, or from public or private research centers.
L'archive ouverte pluridisciplinaire HAL, est destinée au dépôt et à la diffusion de documents scientifiques de niveau recherche, publiés ou non, émanant des établissements d'enseignement et de recherche français ou étrangers, des laboratoires publics ou privés. 


\title{
Review on single track vehicle and motorcycle simulators
}

\author{
L. Nehaoua and H. Arioui and S. Mammar
}

\begin{abstract}
In this paper, we present a review of the existent two-wheeled vehicle simulators. A description of a motorcycle simulator, designed at INRETS-IBISC laboratory is given. Mechatronics aspects and the various consideration to built such a simulation tool will be discussed.

Index Terms-Motorcycle riding simulators.
\end{abstract}

\section{INTRODUCTION}

The human-centered applications are becoming more prevalent in the different science disciplines such as risk prevention, driving and surgical training and anomaly detection (handicap). Especially, the context of road safety is growing to constitutes an essential international issue of research which leads governments in a daily struggle to reduce the number of roadway accidents through prevention and by testing new safety systems and driver assistance.

To achieve that, governments have taken various draconian measures (radar speed checks, speed limitation, etc.). Despite a quite stabilization in the number of serious accident, these solutions often repressive have been shown their limits and so, forcing the competent authorities to change strategy and invest more in searching for more appropriate solutions. Indeed, driver behavior is regarded as a crucial factor in traffic accidents. It is therefore necessary to conduct studies to understand how much a behavior modification may to improve the road safety.

The idea of driving simulation does not start from today. Historically, man has tried to reproduce different contexts within a given environment to address a social or a fictional thought. Driving simulation is often considered as an art than a science which reflects the complexity of the mechanism for creating a virtual environment. In this context, driving simulators have been used extensively in the aerospace and automotive domains. It must be said that this tool has attracted industrial companies and academic communities because of its various skills, namely: formation, prototyping, safety requirements and scenarios reproducibility.

In this paper, we present a review of the existent twowheeled vehicle simulators. Next, a description of a motorcycle simulator, designed at INRETS-IBISC laboratory is given.

\section{PRESENT TWO-WHEELED VEHICLE SIMULATOR}

While enormous means have been invested to improve the comfort and safety of drivers car vehicle, those of the two-wheeled vehicles have been for long time the subject

IBISC Lab., Evry Val d'Essonne University, 40, Rue du Pelvoux, 91020 Evry Cedex, France \{nehaoua, arioui, mammar@ibisc.univevry.fr\} of a secondary priority. In recent years, public awareness and environmental challenges have forced governments to respond.

According to the literature, there are hundreds of car driving simulators throughout the world. Whether academic, industrial or commercial, many institutions have embarked on building their own prototype for different purposes [1], [2]. However, the bibliography of two-wheeled simulators is poor. The main work was done by Japanese and Italian industrial institutions.

\section{A. Reduced motion motorcycle simulator}

The first simulators were in their largely fixed base. A full or reduced version of a two-wheeled vehicle is lay down in front a screen dedicated to the visual environment. Most of these early simulators come from Japan, where driving schools have started to include riding simulation for training.

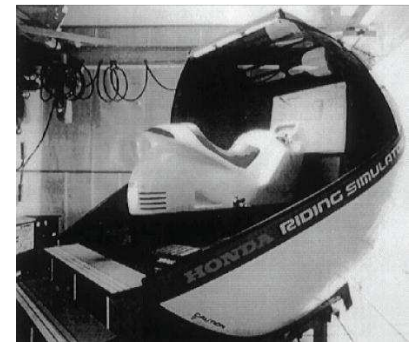

Fig. 1. Honda simulator: First prototype

In 1988, HONDA Corporation started to develop a series of motorcycle simulators. The first prototype of a moving platform simulator was designed to test the feasibility of driving simulation to reproduce the basic maneuvers of motorcycle dynamics behavior (Fig.1). The mobile platform has 7 actuated axis to simulate 4 Degrees of freedom (DOF) including roll, yaw, pitch, and handlebars steer. A cradle mechanism was developed to simulate the feeling of sustained acceleration. To drive this simulator, a linear motorcycle dynamics model is used to compute the dynamic behavior according to the rider's actions. However, it was found that the rider fails to control the simulator while taking a turn because of the absence of centrifugal force, and therefore, the lateral acceleration is different from that experienced in real driving situation. Specifically, on simulator, drivers had a tendency to drive the simulator as a car vehicle. The notion of counter steering is quite hard to reproduce and in many cases the simulator motion became uncontrollable [4], [5].

From these assessments, a second prototype was developed. The main objective of this second generation stay 
unchanged, namely, training for urban traffic situations in a safe environment. Therefore, the controllability of the simulator was of utmost importance than the reproduction of the primary dynamic characteristics of the virtual two-wheeled vehicle. It was important that simulator's user can easily manipulate it and surpass the the first prototype limitations.

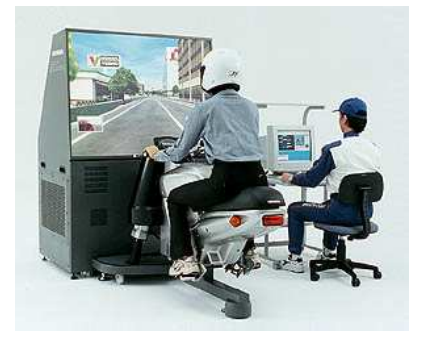

Fig. 2. Honda simulator: Second prototype

In this context, "Easy to manipulate" means that the the simulator's user can easily turn in the desired direction according to the visual scene. Therefore, motorcycle dynamics model was tuned from the performed experiments on different roads and for different maneuvers. The architecture of the new platform was completely changed (Fig.2). The cradle system was removed and only 3DOF have been preserved: pitch $\left( \pm 10^{\circ}\right)$, roll $\left( \pm 15^{\circ}\right)$ and steer $\left( \pm 30^{\circ}\right)$. Next, this prototype was installed in the center of traffic education at Suzuka since 1991 to verify the effectiveness of such a simulator as an approved training tool. This version was marketed in 1996, supported by a Japanese law requiring all candidates to complete off-road training before seeing the grant of a ride license [6].

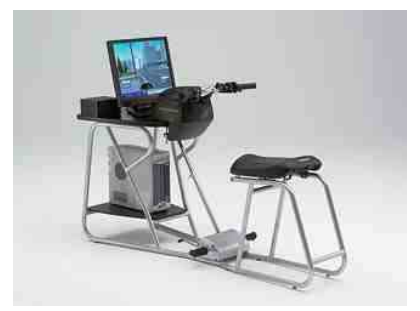

Fig. 3. The Honda SMARTrainer low-cost simulator [7]

Within the same strategy, Honda commercialize its "lowcost" simulator: the SMARTrainer (Fig.3). Physically, it consists of a tubular chassis, a seat, handlebar, control pedals and speed selector. The whole architecture is connected to a computer where a simulation software is installed. The primary mission of this educational tool is to introduce the rider in a given environment. Next, the simulator user engages into a chosen path, makes party from the proposed recommendations and flows through a virtual environment. After a first pass, the student receives an evaluation report that points the different driving errors and the number of incidents or serious accidents.

In the same sense, Kawasaki Heavy Industries was approached by the Japanese National Police Agency to develop motorcycle simulator for riding school training. By using a head mounted display, the rider is able to look side-to-side, use rear view mirrors and lean forward at intersections to check traffic (Fig.4). After simulation, problem areas can be instantly reproduced and viewed from several angles. Otherwise, limited information has been communicated about this simulator.

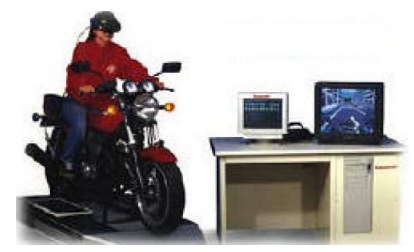

Fig. 4. Kawasaki motorcycle simulator

Besides, other motorcycle simulators was intended to be designed, but it is unclear whether the simulators are developed or not (some patents were disposed). Among them, Dr-Ing Reiner Foerst GmbH company in German has developed a range of simulators including a motorcycle one. It looks like the Kawasaki one but the virtual environment is projected onto a front screen and a roll motion can be done mechanically.

\section{B. Parallel mechanical platform based simulator}

Some two-wheeled simulators were built using a parallel mechanical platform, or the so called, Gough-Stewart. The main advantage of such a platform is the ability to configure exactly an instantaneous center of rotation by a combination of the spatial 6DOF. This property is very important in driving simulation because the position of the pitch, roll and yaw rotation affects directly the quality of the motion cueing and allows avoiding spurious movement due to the coupling between the different degrees of freedom. This fact were extensively exploited by car vehicle companies to design a high level driving simulators.

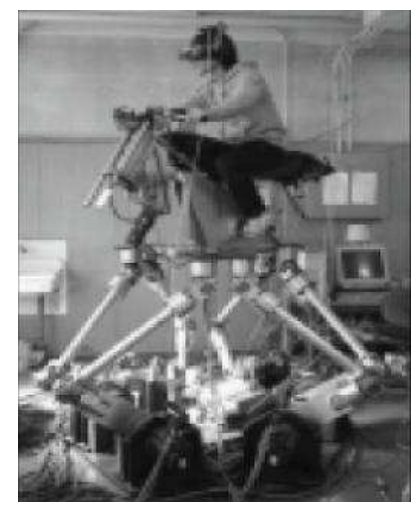

Fig. 5. Parallel platform based motorcycle simulator developed in Japan

In the two-wheeled vehicle simulation field, the first application seems going straightforward from Japan. In this version, a home designed motorcycle cockpit is mounted on a parallel mechanical platform (Fig.5). Herein, the rider also uses a head mounted display for the visual projection. This 
device is also equipped with a lean sensor that measures the spatial position of the driver's head which serves for the correction of the virtual environment with respect to the measured head position [8]. Otherwise, no further informations was published about the effectiveness of such a solution in enhancing the riding simulation.

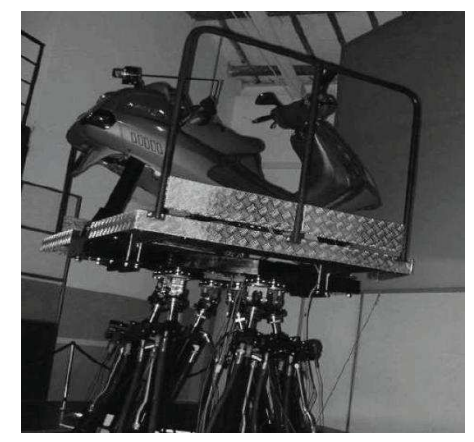

Fig. 6. Moris motorcycle simulator

Outside Japan, a simulator was born from a collaboration between the PERCRO laboratory and motorcycle manufacturer Piaggio [9]. Designed as a rapid prototyping tool, it is based on a 6 DOF mechanical parallel platform hydraulically actuated. A real scooter chassis is mounted on the mobile platform (Fig.6). The main user actions are instrumented with haptic devices, and a DC motor was added to reproduce the engine vibration. The handlebar is equipped with a brushless servo-motor generating a maximum torque of 15(N.m) for the force feedback. The overall architecture is organized around several modules communicating via an optical and ISA (Industry Standard Architecture) buses. A computer system based on VxWorks is implemented for the real-time synchronization of the various modules.

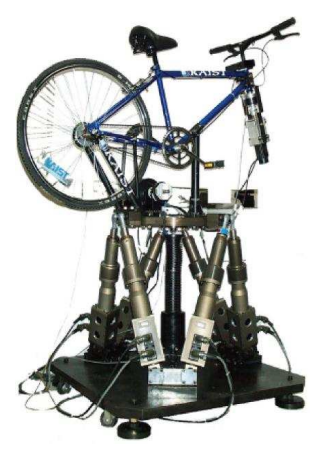

Fig. 7. KAIST bicycle simulator

In the same way, a bicycle simulator was built at KAIST (Korean Advanced Institute of Sciences and Technologies) [10]. The motion generation is ensured by a 6DOF GoughStewart platform electrically actuated, on which a bicycle frame is fixed (Fig.7). The handlebar and the pedal are respectively equipped with haptic devices. In one hand, the bicycle handlebar and the corresponding DC motor are directly connected via magneto-rheological damper. On the other hand, the cycle-pedal and the corresponding AC motor are connected via a magneto-rheological brake. These configuration aims to create the resisting forces due to air resistance, roadway friction and the resistive gravity force due to the road banking [11].

\section{Serial mechanical platform based simulators}

Based on a serial mechanical architecture, a riding simulator is designed at the Department of Mechanical Engineering at Padua University [12]. This simulator allows the simulation of 5DOF including roll, pitch, yaw, lateral displacement and steer angle (Fig.8). The mechanical structure consists of a cubic cage that supports the frame motion via four steel suspended cables. This solution is supposed to reduce the frictional forces and allow an optimal distribution of the gravity forces. The main frame of the simulator has $2 \mathrm{DOF}$ with respect to the cage, i.e, lateral displacement and yaw rotation. A cradle is attached to the main frame for roll degree of freedom. A saddle is mounted on the cradle with another degree of freedom, which corresponds to the pitch.

In this simulator, all rider actions, except rider lean, are instrumented with adequate sensors and signal acquisition is performed by DAC National Instruments solution. A virtual motorcycle dynamics, namely FastBike code, is used to drive the virtual motorcycle. FastBike is based on multibody formulation with an enhanced tire modeling including carcass deformation and allowing the simulation of 11DOF [13]. To drive this model, the rider torque excreted on the handlebar is measured by a home developed torque sensor.

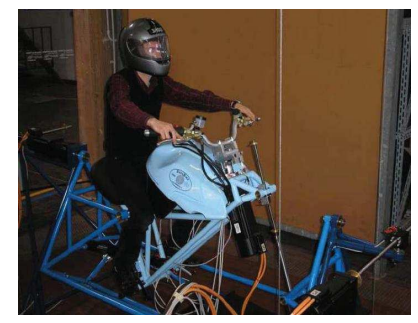

Fig. 8. The UNIPD simulator at Padua

Several studies for rider safety, as collision warning and rider fall, were carried out using these simulator [14]. Also, a validation work for a bio-mechanical rider model is done [15].

\section{INRETS-IBISC MOTORCYCLE SIMULATOR}

Within the framework of the SIMACOM project, a riding motorcycle simulator was conceived in collaboration between the INRETS and IBISC Laboratories in France [3] (Fig.9).

In this section, we discuss the objectives of designing such a tool, the different consideration to be taken into account and the simulator's description.

\section{A. Aims and design considerations}

While driving car or two-wheeled vehicles, informations sources that allow the roadway tracking and to the vehicle control are various. The simulation aim is to stimulate the 
driver sensor to give him some illusion of a driving on a real vehicle. This illusion is a complex phenomenon which involves the human proprioceptive sensors in particular the visual, kinesthetic and vestibular ones.

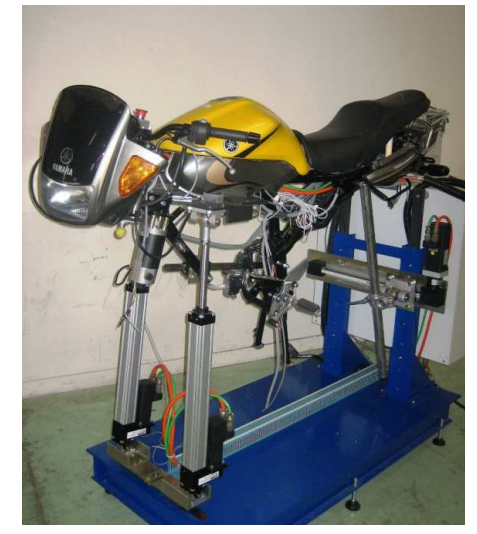

Fig. 9. The developed riding motorcycle simulator by INRETS-UEVE

From the previous section, it can be seen that the simplest way to build a simulator is to adopt for a parallel mechanical platform with 6DOF. Nevertheless, despite its advantages, their cost is far from being affordable to be supported by various interested operators.

The choice of the mechanical architecture is guided by the final needs which require ensuring an acceptable and defined level of perception. The present simulator is intended for two specified tasks, namely, risk training by prevention and awareness of dangerous riding situations, and behavior observation in normal riding situations.

To define the simulator's platform kinematics, it is therefore prerequisite to establish the different scenarios to be simulated, namely:

- Risk training: the purpose of the risk training is to identify, a priori, hazardous situations. On motorcycle, some situations could not be overtaken, as front wheel skid. In such a case, the transient time between stability and instability is very short, therefore, the reproduction of this behavior involves highly actuation performance. Nevertheless, the simulator would be still able to inform the users that he is going into an hazardous situations.

- Behavior observation: herein, the purpose is to enable researchers to understand the various cues perceived by the rider. These studies are important for the development of driver assistance systems. Limit driving situation should therefore be reproduced as well as normal driving ones.

For both scenarios, putting into a target situation should be preferred to faithful of the full scale motion reproduction, and hence, the final simulator's architecture (workspace, actuators' performance, etc.) should go in this direction. This idea is also compatible with the development of a low-cost marketable tool. From these considerations, the chosen DOF of motion are:

- Roll: it is the most important movement in the motorcycle dynamics. This DOF is essential for the stabilization and control of the vehicle. It is mainly involved in the simulation of cornering, slalom and lane change maneuvers. On simulator, this motion is rendered by combining the platform roll with a visual one. For example, a roll of about $\pm 20^{\circ}$ is used to simulate the rider fall.

- Pitch: This DOF is used for the restitution of a part of the longitudinal acceleration, braking or fork suspension.

- Yaw: This motion has been specifically selected to reproduce the rear wheel skidding as in classical risky situations. However, front wheel skidding will not be retained because it is immediately fatal, and so, it is useless to propose a recovery technique that is very difficult in a real case.

On the other hand, the multiplication of stimulation devices greatly enhance the simulation fidelity. For this, a double force feedback is implemented on the handlebars. The first one is used to create an inertial delay on rider bust during acceleration and braking. Thus, an effort is created on rider's arms by varying the distance between the saddle and the handlebar. On the other hand, the second one is intended for the torque feedback resulting from tire-road interaction.

The position of the different rotations axes is of great importance. To our knowledge, no psychophysical study has been conducted, except in some special cases [6]. Therefore, these axes are defined from the kinematics of a real motorcycle. To simulate yaw motion, a slide is attached to the rear frame of the simulator (Fig.10). The roll axis is taken in the plane of symmetry of the motorcycle with an adjustable height to test several configurations. Finally, the pitch axis is considered regarding the displacement of the front fork during acceleration and braking, therefore, this axis passes through the back of the motorcycle.

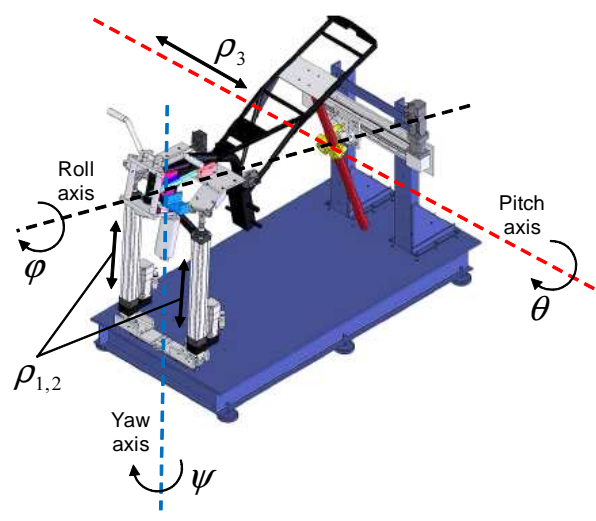

Fig. 10. CAD model of the mechanical platform with the rotation axes

Experimental studies have shown that the closed loop bandwidth at $-3 \mathrm{~dB}$ is respectively $11.8(\mathrm{rad} / \mathrm{s})$ for roll and pitch motion and $17.2(\mathrm{rad} / \mathrm{s})$ for the yaw, ie, $1.91(\mathrm{~Hz})$ and $2.73(\mathrm{~Hz})$. Compared to 3-4 $(\mathrm{Hz})$ observed in a real motorcycle dynamics, one may concludes that the simulator's platform has limited features for the restitution of transient accelerations (emergency braking, sudden acceleration). 
Nevertheless, this limitation is not crippling insofar as the driving simulation is based on illusion generation and not on the full scale motion cueing. Hence, we can affirm that the dynamic characteristics of the current platform allow us to sufficiently stimulate the driver proprioceptive sensors, but only a psychophysical experiment should certified such an assessment.

\section{B. Motion cueing algorithms}

The objective of a motion cueing system, when used jointly with a visual system, is to stimulate the rider's perceptual modalities so he can feel sufficient cues experimented in a real driving environment. So, cues restitution is considered as the central element in driving simulation. However, the real movement generation is impossible because of the inherent limitations of mechanical platform. It is therefore necessary to develop techniques that overcome these limitations and make motion occurring only into the feasible workspace. It is the role of the motion cueing algorithms (MCA), also called washout filters.

The basic idea of the MCA is to achieve a frequency separation, through dedicated filters, to retrieve transient and sustained acceleration where the transient one can be directly rendered. The differences between the various proposed algorithms lie in these filters synthesis and tuning [16].

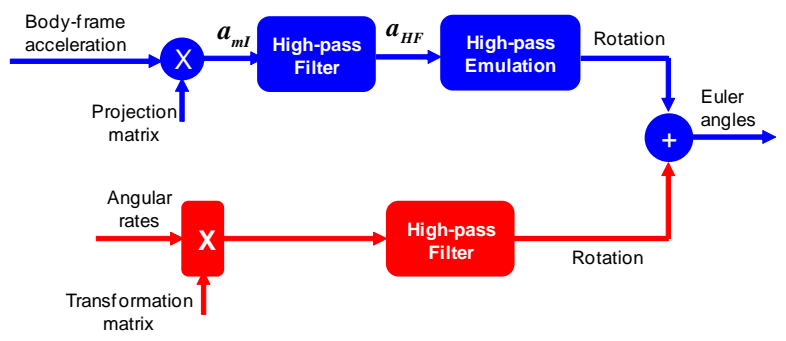

Fig. 11. Emulation of a transient acceleration by a only a rotation

The current mechanical architecture of the simulator provides no translational motion, therefore, rotations will be exploited to reproduce linear accelerations as suggested in [17]. In this scheme, the body-fixed linear acceleration $a_{m}$ is projected into the inertial reference frame to get $a_{m I}$ (Fig.11). Next, by high-pass filtering, one obtains transient part $a_{H F}$ which passes though a bloc named High-pass Emulation. Emulation is a simple kinematic principle of the circular motion. Indeed, the linear acceleration to be emulated can be split into two components, one radial and one tangential. If the first component can't be rendered, the second one is used to calculate an angular acceleration, and thus, a double integration yields the vector of Euler angles.

Figures (12) presents the performance of the HP emulation to generate a linear acceleration of type square. It may be seen that in addition to the ability of this approach to reproduce a part of the transient component, it can also render a part of the sustained acceleration. Therefore, the low-frequency channel, present in the MCA original version, is no longer necessary. Moreover, the need for tilt is less essential. However, the plot corresponding to the angular rates highlights the limits of this method, especially, for large transient acceleration (significant acceleration and emergency braking). In other words, if the amplitude of the transient component of the linear acceleration is too large, the tilt platform rotation is faster and thus detectable by the semicircular canals (where the angular rates threshold is reported to be $\left.\left(3.7^{\circ} / \mathrm{s}\right)[18]\right)$. This compromise shows that a satisfactory linear acceleration cueing can be guaranteed only by a linear translation.
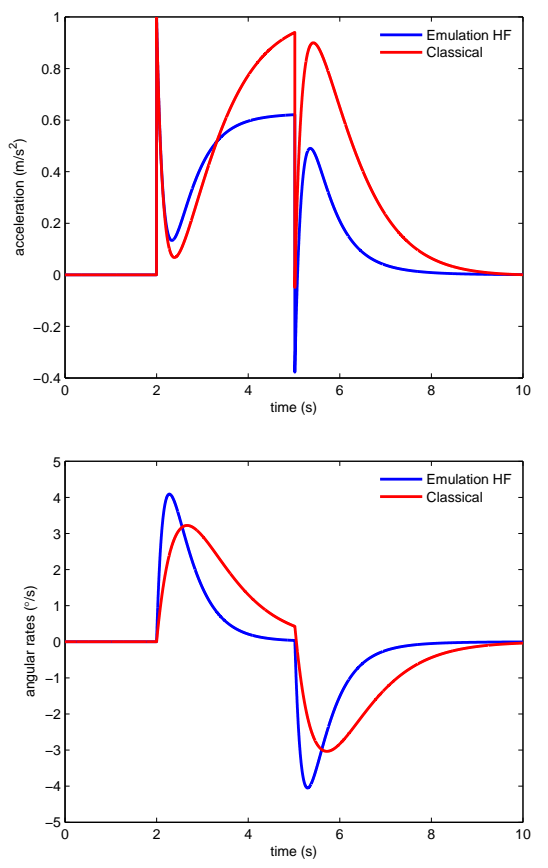

Fig. 12. Performance of the HP emulation to generate a linear acceleration

\section{Platform mechatronics}

Figure (13) shows the data flow involving different exchanged variables and also, the communication modes between the various simulator's modules. The core of this architecture is the real-time kernel that facilitates the lowlevel management. By analyzing this diagram, we can pickup a first glimpse on the tasks sequencing, as following:

- After initialization, the rider actions are acquired via the CAN bus at 500(Hz) and sent to "xPC Target" PC. Next, this acquisition is used to solve the virtual motorcycle states.

- On the one hand, virtual motorcycle states as speed, longitudinal and lateral positions, roll, pitch and yaw angles are sent to the visual PC via UDP (User Datagram Protocol) to allow the scene projection. These data are sent at a frequency, minimally, equal to the refresh rate of the visual projection.

- On the other hand, the platform reference trajectories as calculated by MCA and inverse kinematics algorithms are transmitted to simulator servo-drives by CAN bus at a rate of $100(\mathrm{~Hz})$. 


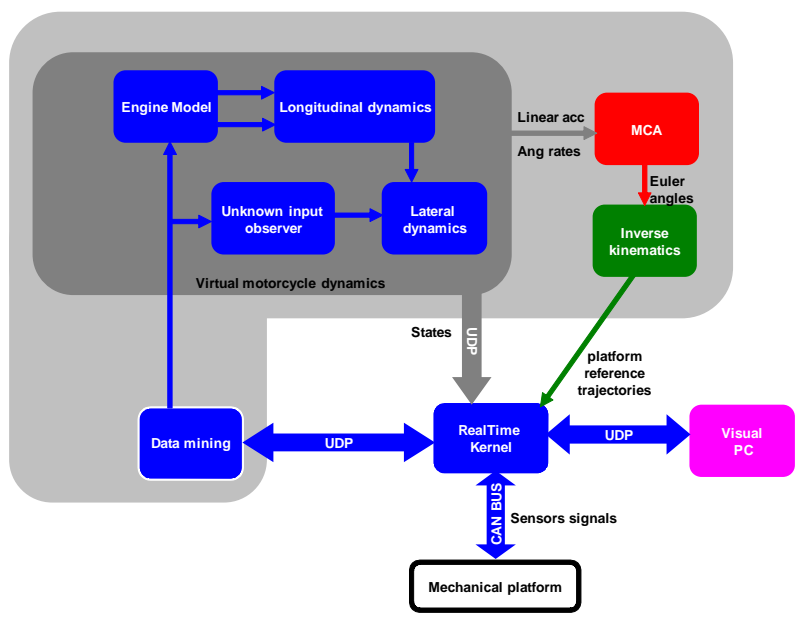

Fig. 13. Mechatronics data exchange

Signal acquisition is ensured by home electronic card based on a NEC-V853 micro-controller. This card has 12 AI, $16 \mathrm{AO}$ as well as multiple digital I/O. The embedded FPGA allows the management of 24 buffered binary inputs dedicated to the acquisition of optical encoders signals. In the other hand, the simulator's mechanics is electrically actuated via 3 servo-drives type Lust-CDD3000. Finally, the steering haptic feedback is motorized through a DC motor type Parvex620.

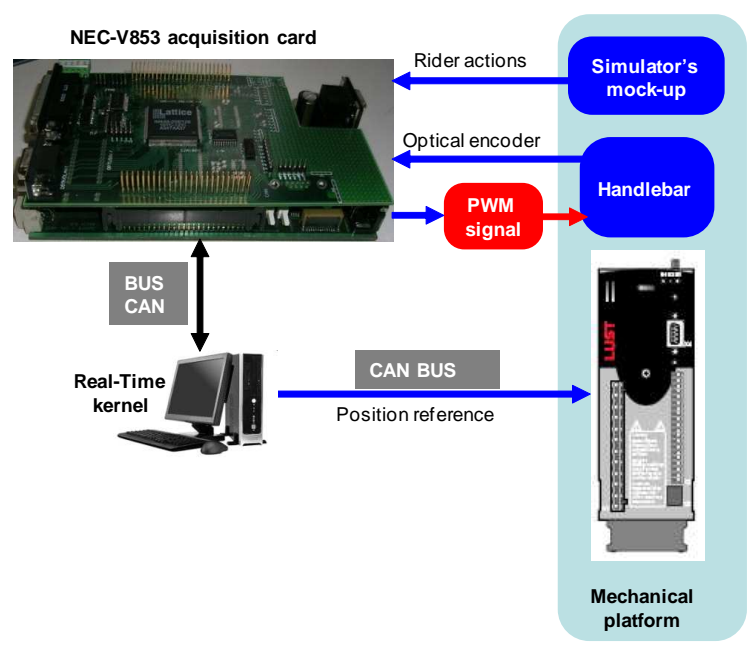

Fig. 14. Control scheme and acquisition mode

Initially, the servo-drives were driven in speed control loop [19]. This control mode requires the position measurement which generates delays, very undesirable in driving simulation. Therefore, the servo-drive are directly controlled in position without an external control-loop (Fig.14). Trajectory tracking is performed by optimizing the in-situ servo-drive inner control-loop. This solution seems to increase greatly the performance and eliminates the delay problem.

\section{CONCLUSiON}

This paper presents a literature review on the existing twowheeled simulators. Next, a description of the motorcycle simulator developed at INRETS-IBISC is described. The different consideration to be taken into account in designing such a simulator are discussed and the adopted mechanics is detailed. Finally, a brief presentation focuses on the software architecture and the simulator's mechatronics is given. The electronic instrumentation, tasks synchronization and realtime aspects are presented.

\section{REFERENCES}

[1] J.S. Freeman, G.W. Papelis, Y. Papelis, A. Tayyab, R.A. Romano and J.C. Kuhl, "The Iowa driving simulator : An implementation and application overview”, SAE Technical Paper Series,n (950174), 1995.

[2] L. Nehaoua, H. Mohellebi, A. Amouri, H. Arioui, S. Espie and A. Kheddar, "Design and control of a small-clearance driving simulator", IEEE Transactions on Vehicular Technology, vol. 57(1), pp 736-746, 2008.

[3] H. Arioui, L. Nehaoua, S. Hima, N. Séguy, and S. Espié, "Mechatronics, Design, and Modeling of a Motorcycle Riding Simulator", IEEE/ASME Transactions on Mechatronics, vol. 15(5), pp 805-818, 2010.

[4] Y. Miyamaru, G. Yamasaky and K. Aoky, "Development of motorcycle riding simulator and its prehistory", JSME Review, vol. 50, 2000.

[5] Y. Miyamaru, G. Yamasaky and K. Aoky, "Development of motorcycle riding simulator", JSAE Review, vol. 23, pp 121-126, 2002.

[6] G. Yamasaky, K. Aoky, Y. Miyamaru and K. Ohnuma, 'Development of motorcycle training simulator", JSAE Review, vol. 19, pp 81-85, 1998;

[7] url = "http://world.honda.com"

[8] S. Chiyoda, K. Yoshimoto, D. Kawasaki, Y. Murakami and T. Sugimoto, "Development of a motorcycle simulator using parallel manipulator and head mounted display, Proceedings of Driving Simulation Conference DSC, 2000.

[9] D. Ferrazzin, F. Barbagli, C.A. Avizzano, D. Di Pietro and M. Bergamasco, "Designing new commercial motorcycles through a highly reconfigurable virtual reality-based simulator", Advanced Robotics, vol. 17(4), pp 293-318, 2003.

[10] D.S. Kwon et al, "Kaist interactive bicycle simulator", Proceedings of IEEE International Conference on Robotics and Automation (ICRA01), pp 2313-2318, Seoul, Korea, 2001.

[11] J.C Shin and C.W. Lee, "Riders net moment estimation using control force of motion system for bicycle simulator", Journal of Robotics Systems, vol. 21(11), pp 597-607, 2004.

[12] V. Cossalter, A. Doria and R. Lot, "Development and validation of a motorcycle riding simulator", World Automotive Congress FISITA, Barcelona, Spain, 2004.

[13] V. Cossalter and R. Lot, "A motorcycle multibody model for real time simulation based on the natural coordinates approach", Vehicle System Dynamics, vol. 37(6), pp 423-447, 2002.

[14] V. Cossalter, R. Lot, M. Massaro and R. Sartori, ”Development and Testing of Assistant Rider Systems with the UNIPD Motorcycle Riding Simulator", XIX Congresso Aimeta Associazone Italiana di Meccanica Teorica e Applicata, Italy, 2009.

[15] V. Cossalter, A. Doria, D. Fabris and M.Maso, "Measurement and identification of the vibrational characteristics of motorcycle riders", International Conference on Noise and Vibration Engineering conference, Belgium, 2006.

[16] M.A. Nahon and L.D. Reid, "Simulator motion-drive algorithms : A designers perspective", Journal of Aircraft, vol. 13(2), pp 356-362, 1990.

[17] N.A. Pouliot, C.M. Gosselin and M.A. Nahon, "Motion simulation capabilities of 3DOF flight simulators", Journal of Aircraft, vol. 35(1), pp 917, 1998.

[18] A.J. Benson, "Perception and control of self motion, chapitre Sensory functions and limitations of the vestibular systems", pp 145-170, laurence erlbaum ass edition, 1990.

[19] L. Nehaoua, S. Hima, H. Arioui, N. Séguy and S. Espié, "Design and Modeling of a New Motorcycle Riding Simulator", Proceedings of 2007 American Control Conference (ACC07), pp 176-181, New York City, USA, 2007. 